\title{
\begin{tabular}{l|l} 
Mibraries & DSpace@MIT
\end{tabular}
}

\author{
MIT Open Access Articles
}

\section{Qubit Protection in Nuclear-Spin Quantum Dot Memories}

The MIT Faculty has made this article openly available. Please share how this access benefits you. Your story matters.

Citation: Kurucz, Z. et al. "Qubit Protection in Nuclear-Spin Quantum Dot Memories." Physical Review Letters 103.1 (2009): 010502. (c) 2009 The American Physical Society.

As Published: http://dx.doi.org/10.1103/PhysRevLett.103.010502

Publisher: American Physical Society

Persistent URL: http://hdl.handle.net/1721.1/51773

Version: Final published version: final published article, as it appeared in a journal, conference proceedings, or other formally published context

Terms of Use: Article is made available in accordance with the publisher's policy and may be subject to US copyright law. Please refer to the publisher's site for terms of use. 


\title{
Qubit Protection in Nuclear-Spin Quantum Dot Memories
}

\author{
Z. Kurucz, ${ }^{1,2, *}$ M. W. Sørensen, ${ }^{3}$ J. M. Taylor, ${ }^{4,5}$ M. D. Lukin, ${ }^{5}$ and M. Fleischhauer ${ }^{1}$ \\ ${ }^{1}$ Fachbereich Physik, University of Kaiserslautern, D-67663 Kaiserslautern, Germany \\ ${ }^{2}$ Research Institute for Solid State Physics and Optics, H.A.S., H-1525 Budapest, Hungary \\ ${ }^{3}$ Niels Bohr Institute, University of Copenhagen, DK-2100 Copenhagen, Denmark \\ ${ }^{4}$ Department of Physics, Massachusetts Institute of Technology, Cambridge, Massachusetts 02139, USA \\ ${ }^{5}$ Department of Physics, Harvard University, Cambridge, Massachusetts 02138, USA
}

(Received 13 March 2009; published 2 July 2009)

\begin{abstract}
We present a mechanism to protect quantum information stored in an ensemble of nuclear spins in a semiconductor quantum dot. When the dot is charged the nuclei interact with the spin of the excess electron through the hyperfine coupling. If this coupling is made off-resonant, it leads to an energy gap between the collective storage states and all other states. We show that the energy gap protects the quantum memory from local spin-flip and spin-dephasing noise. Effects of nonperfect initial spin polarization and inhomogeneous hyperfine coupling are discussed.
\end{abstract}

DOI: 10.1103/PhysRevLett.103.010502

An essential ingredient for quantum computation and long-distance quantum communication is a reliable quantum memory. Nuclear spins in semiconductor nanostructures are excellent candidates for this task. With a magneton 3 orders of magnitude weaker than electron spins, they are largely decoupled from their environment, and the hyperfine interaction with electron spins allows one to access ensembles of nuclear spins in a controlled way [1-10]. In particular, the quantum state of an electron spin can be mapped onto the nuclear spins, giving rise to a longterm memory [3-7]. Nevertheless, memory lifetimes are limited, e.g., by dipole-dipole interactions among the nuclei. In this Letter, we demonstrate that the presence of the electron spin in the quantum dot substantially reduces the decoherence of this collective memory associated with surrounding nuclear spins. The virtual transitions between electronic and nuclear states can be used to produce an energy shift proportional to the number of excitations in the storage spin-wave mode. This isolates the storage states energetically and protects them against nuclear-spin flips and spin diffusion.

Consider a quantum dot charged with a single excess electron as indicated in Fig. 1. The electron spin $\hat{\mathbf{S}}$ is coupled to the ensemble of underlying nuclear spins $\hat{\mathbf{I}}^{j}$ by the Fermi contact interaction,

$$
\hat{H}_{\mathrm{hf}}=\mathcal{A} \sum_{j}^{N} \varrho_{j}\left[\hat{I}_{z}^{j} \hat{S}_{z}+\frac{1}{2}\left(\hat{I}_{+}^{j} \hat{S}_{-}+\hat{I}_{-}^{j} \hat{S}_{+}\right)\right],
$$

where $\mathcal{A}$ is the average hyperfine interaction constant, $\mathcal{A} \approx 90 \mu \mathrm{eV}$ for $\mathrm{GaAs}$, and $\varrho_{j}$ is proportional to the electron density at the position of the $j$ th nucleus, $\sum_{j} \varrho_{j}=$ 1. For convenience, we introduce the collective operators $\hat{\mathbf{A}} \equiv \sum_{j} \varrho_{j} \hat{\mathbf{I}}^{j}$. The first term in Eq. (1) yields the Overhauser field, an effective magnetic field for the electron, and also the Knight shift for each nuclei. The flip-flop terms in Eq. (1), $\hat{H}_{\mathrm{JC}}=\frac{\mathcal{A}}{2}\left(\hat{A}_{+} \hat{S}_{-}+\hat{A}_{-} \hat{S}_{+}\right)$, can be used to
PACS numbers: 03.67.Pp, 03.65.Yz, 73.21.La, 76.70.-r

polarize the nuclear spins [1,2], and to map the electron's spin state into a collective spin mode of the nuclei [3-5]. As will be shown here, the same can be used to provide a protective energy gap.

Fully polarized nuclei.-We start by reconsidering the storage of a qubit in collective nuclear states [3]. In the case when all the nuclear spins are initially polarized in the $-z$ direction (zero temperature limit), the $|\downarrow\rangle_{e}$ and $|\uparrow\rangle_{e}$ spin states of the electron are mapped onto the nuclear-spin states $|\mathbf{0}\rangle \equiv|-I,-I, \ldots,-I\rangle$ and

$$
|\mathbf{1}\rangle \equiv \frac{\mathcal{A}}{\Omega} \hat{A}_{+}|\mathbf{0}\rangle \propto \sum_{j} \varrho_{j}\left|-I, \ldots,(-I+1)_{j}, \ldots,-I\right\rangle,
$$

respectively. $\hat{H}_{\mathrm{JC}}$ couples the state $|\mathbf{0}\rangle|\uparrow\rangle_{e}$ to $|\mathbf{1}\rangle|\downarrow\rangle_{e}$ with an angular frequency $\Omega=\mathcal{A}\left(\sum_{j} \varrho_{j}^{2} 2 I\right)^{1 / 2}$. The detuning between these two states, $\delta=\delta^{\mathrm{OH}}+\delta^{\mathrm{el}}$, comes from the Overhauser field, $\delta^{\mathrm{OH}}=-\mathcal{A} I$, and from the electron's intrinsic energy splitting $\delta^{\text {el }}$ due to, e.g., an external magnetic field or a spin-state dependent Stark laser pulse [11]. Coherent flip-flops between the electron and nuclear spins can be brought into resonance $(\delta \ll \Omega)$ through $\delta^{\text {el }}$. Then $|\mathbf{0}\rangle\left(\alpha|\downarrow\rangle_{e}+\beta|\uparrow\rangle_{e}\right)$ is rotated to $(\alpha|\mathbf{0}\rangle+\beta|\mathbf{1}\rangle)|\downarrow\rangle_{e}$, and the

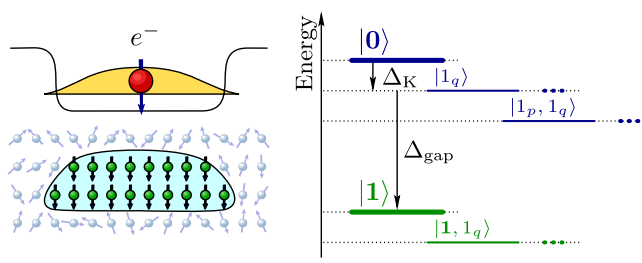

FIG. 1 (color online). Left: Charged quantum dot with a single, polarized excess electron. Right: Spectrum of the effective nuclear Hamiltonian in the presence of a polarized electron. Off-resonant hyperfine coupling results in a gap $\Delta_{\text {gap }}$ between the storage state $|\mathbf{1}\rangle$ and the nonstorage states $\left|1_{q}\right\rangle . \Delta_{\mathrm{K}}$ denotes the Zeeman shift due to the effective magnetic field associated with the electron spin (Knight shift). 
quantum information is transferred from the electron to the nuclear-spin ensemble and back $[3,4]$.

Assume that, after the qubit has been written into the nuclei, the polarized electron is not removed from the dot but the hyperfine flipflops are tuned off-resonant $(\delta \gg \Omega)$. Now, real transitions can no longer take place between $|\mathbf{1}\rangle|\downarrow\rangle_{e}$ and $|\mathbf{0}\rangle|\uparrow\rangle_{e}$. However, the residual virtual transitions repel the two states from each other, in analogy to the dynamic Stark effect. As a result, after adiabatic elimination of the electronic states, the energy of state $|\mathbf{1}\rangle$ gets shifted by $\Delta_{\text {gap }}=-\Omega^{2} / 4 \delta$. The other, orthogonal states also having exactly one spin flipped (denoted by $\left|1_{q}\right\rangle$ in Fig. 1) are "subradiant," i.e., are not coupled via $\hat{H}_{\text {JC }}$ to the electron. Therefore, they are unaffected by the shift. This is the origin of the energy gap.

To understand the protection scheme, let us introduce nuclear-spin waves. For highly polarized nuclei, one can introduce bosonic operators through the HolsteinPrimakoff transformation: $\hat{a}_{j} \approx \hat{I}_{-}^{j} / \sqrt{2 I}$ and $\hat{a}_{j}^{\dagger} \hat{a}_{j}=\hat{I}_{z}^{j}+$ $I$. This allows us to define the bosonic spin waves

$$
\hat{\Phi}_{q} \equiv \sum_{j} \eta_{q j} \hat{a}_{j}, \quad \hat{\Phi}_{q}^{\dagger} \equiv \sum_{j} \eta_{q j}^{*} \hat{a}_{j}^{\dagger},
$$

where the unitary matrix $\eta_{q j}$ describes the mode functions. We identify the storage mode $q=0$ as the one given by $\eta_{0 j}=\sqrt{2 I} \frac{\mathcal{A}}{\Omega} \varrho_{j}$, and write $|\mathbf{1}\rangle=\hat{\Phi}_{0}^{\dagger}|\mathbf{0}\rangle$. This is the mode which is directly coupled to the electron spin. In fact, $\hat{H}_{\mathrm{JC}} \approx \frac{\Omega}{2}\left(\hat{\Phi}_{0}^{\dagger} \hat{S}_{-}+\hat{\Phi}_{0} \hat{S}_{+}\right)$is a Jaynes-Cummings coupling in the bosonic approximation. After adiabatically eliminating the electronic states, $\hat{H}_{\mathrm{JC}}$ reduces to $\hat{H}_{\text {gap }}=$ $-\frac{\mathcal{A}^{2}}{4 \delta} \hat{A}_{+} \hat{A}_{-} \approx \Delta_{\text {gap }} \hat{\Phi}_{0}^{\dagger} \hat{\Phi}_{0}$. As shown in Fig. 1, $\hat{H}_{\text {gap }}$ lifts the degeneracy between states of different number of storage-mode excitations. This is the key feature of our protection scheme: any decoherence process that is associated with a transition from the storage mode $\hat{\Phi}_{0}$ to any other mode $\hat{\Phi}_{q}$ now has to bridge an energy difference. If this gap is larger than the spectral width of the noise, the effect of the noise is substantially reduced.

A more detailed analysis shows that the off-resonant interaction with the electron spin-which itself is coupled, e.g., to phonons-leads in general also to an additional decoherence mechanism for the nuclear spins. If the corresponding electron spin dephasing rate $\gamma$ is small compared to the electron's precession frequency $\delta$, the decay rate for the storage mode is reduced by the low probability of exciting the electron spin state: $\gamma \Omega^{2} / \delta^{2} \ll \gamma$.

In addition to the gap, the electron is also responsible for the Knight shift $\hat{H}_{\mathrm{K}}=\mathcal{A} \hat{A}_{z}\left\langle\hat{S}_{z}\right\rangle$. The relative shift between the $|\mathbf{0}\rangle$ and $|\mathbf{1}\rangle$ states, $\Delta_{\mathrm{K}}=-\frac{\mathcal{A}}{2} \sum_{j} \varrho_{j}^{3} / \sum_{j} \varrho_{j}^{2}$, is typically much less than $\Delta_{\text {gap }}$. When the hyperfine coupling is inhomogeneous, however, $|\mathbf{1}\rangle$ fails to be eigenstate of $\hat{H}_{\mathrm{K}}$. Instead, $\hat{H}_{\mathrm{K}}|\mathbf{1}\rangle=\left(-\frac{1}{2} \delta^{\mathrm{OH}}+\Delta_{\mathrm{K}}\right)|\mathbf{1}\rangle+\zeta\left|1^{\perp}\right\rangle$, where $\left|1^{\perp}\right\rangle$ is orthonormal to $|\mathbf{1}\rangle$ and $\zeta^{2}=$ $\frac{\mathcal{A}^{2}}{4} \sum_{j} \varrho_{j}^{4} / \sum_{j} \varrho_{j}^{2}-\Delta_{\mathrm{K}}^{2}$ characterizes the inhomogeneities. Thus, the storage mode is only an approximate eigenmode, and it gradually mixes with nonstorage modes as time passes. This causes loss of the stored qubit. $\left|1^{\perp}\right\rangle$ is, however, off-resonant due to the energy gap, and the corresponding probability of finding the system in state $\left|1^{\perp}\right\rangle$ is bounded by $4 \zeta^{2} / \Delta_{\text {gap }}^{2}$, so the detrimental effect of the inhomogeneous Knight shift is suppressed by the energy gap. In addition, since the admixture of $\left|1^{\perp}\right\rangle$ is a coherent process, it can be cancelled by refocusing (echo) methods.

A larger gap can be achieved by bringing the hyperfine interaction closer to resonance. A nonzero external magnetic field or laser induced ac Stark shifts [11] can partially cancel the Overhauser field, such that $\delta \ll \delta^{\mathrm{el}} \approx-\delta^{\mathrm{OH}}=$ $\mathcal{A} I$. (Of course, $\delta$ should be kept sufficiently large so that the hyperfine coupling remains off-resonant). The requirement of separation of time scales implies $\zeta \ll\left|\Delta_{\text {gap }}\right| \ll$ $\Omega \ll|\delta|$. To estimate the orders of magnitude of the different energies, we take an oblate Gaussian electron density of ratio $(1,1,1 / 3)$ and spin- $\frac{1}{2}$ nuclei. Then we find that $\Delta_{\mathrm{K}}$ and $\zeta$ are inversely proportional to the number of nuclei $N$, whereas $\Omega, \Delta_{\text {gap }} \propto N^{-1 / 2}$ only [Fig. 2(a)].

To analyze the decoherence suppression, we first consider a simplistic noise model where the nuclear spins are coupled to fluctuating, classical fields. The corresponding interaction Hamiltonian is $\hat{V}=\sum_{j} \mathbf{B}^{j} \cdot \hat{\mathbf{I}}^{j}$. We assume isotropic Gaussian noise with zero mean and $\overline{B_{\mu}^{j}(t) B_{\nu}^{k}\left(t^{\prime}\right)}=$ $\delta_{\mu \nu} \xi_{j k} C e^{-\Gamma\left|t-t^{\prime}\right|}$ for $\mu, \nu=x, y, z$, where $\xi_{j k}$ specifies the spatial correlations of the noise acting on different nuclei. For simplicity, the noise spectrum is assumed to be Lorentzian with a width $\Gamma$, although similar results hold for other spectra with high-frequency cutoff.

Let us first discuss the dephasing part of the noise,

$$
\hat{V}_{z}=\sum_{j} B_{z}^{j} \hat{a}_{j}^{\dagger} \hat{a}_{j}=\sum_{p q}\left(\sum_{j} B_{z}^{j} \eta_{p j}^{*} \eta_{q j}\right) \hat{\Phi}_{p}^{\dagger} \hat{\Phi}_{q}
$$

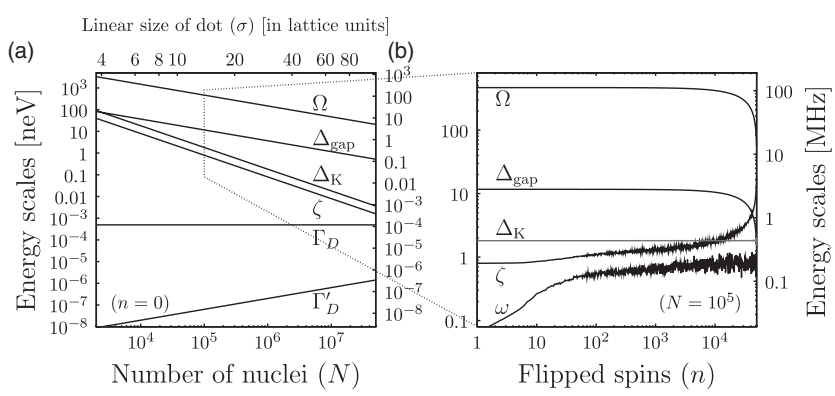

FIG. 2. Hyperfine Rabi frequency $(\Omega)$, protective energy gap $\left(\Delta_{\text {gap }}\right)$, Knight shift difference between the logical states $\left(\Delta_{\mathrm{K}}\right)$, symmetry breaking couplings due to inhomogeneities ( $\zeta$ and $\omega$ ), and qubit decoherence rates due to dipolar spin diffusion without $\left(\Gamma_{D}\right)$ and with $\left(\Gamma_{D}^{\prime}\right)$ protection. (a) The fully polarized (zero temperature) case shown versus the number of spin- $\frac{1}{2}$ nuclei $(N)$ taking part in the storage, i.e., located within $3 \sigma$ of the oblate Gaussian electron distribution with in-plane variance $\sigma$. (b) Estimated energies in dark states $\left|\mathcal{D}_{n, \beta}\right\rangle$ with $n$ spins flipped from the fully polarized state for $N=10^{5}$. Energy units are chosen to match GaAs. $\Delta_{\text {gap }}$ is obtained by taking $\delta=10 \Omega$. 
expressed by the bosonic spin-wave operators (3). Dephasing of individual nuclear spins means transfer of excitations between different spin-wave modes. Especially, it leads to both real and virtual transitions from $|\mathbf{1}\rangle$ to a nonstorage state $\left|1_{q}\right\rangle$. As the latter state is "subradiant" and, thus, equivalent to $|\mathbf{0}\rangle$ when the memory is read out, this process essentially results in damping (for real transitions) and dephasing (for virtual transitions) of the stored logical qubit [12]. Assuming the zero temperature limit with all nonstorage modes $\hat{\Phi}_{q \neq 0}$ in the vacuum state and formally eliminating them in the Markov approximation together with the classical fields, we derive a master equation for the storage mode: $\frac{d}{d t} \hat{\rho}=i\left[\hat{\rho}, E_{z} \hat{\Phi}_{0}^{\dagger} \hat{\Phi}_{0}\right]+$ $\mathcal{L}_{z}(\hat{\rho})$, with energy shift $E_{z}=(1-\Xi) C \Delta_{\text {gap }} /\left(\Gamma^{2}+\right.$ $\left.\Delta_{\text {gap }}^{2}\right)$ and

$$
\begin{aligned}
\mathcal{L}_{z}(\hat{\rho})= & \gamma_{1}\left(2 \hat{\Phi}_{0} \hat{\rho} \hat{\Phi}_{0}^{\dagger}-\hat{\Phi}_{0}^{\dagger} \hat{\Phi}_{0} \hat{\rho}-\hat{\rho} \hat{\Phi}_{0}^{\dagger} \hat{\Phi}_{0}\right) \\
& +\gamma_{2}\left(2 \hat{\Phi}_{0}^{\dagger} \hat{\Phi}_{0} \hat{\rho} \hat{\Phi}_{0}^{\dagger} \hat{\Phi}_{0}-\hat{\Phi}_{0}^{\dagger} \hat{\Phi}_{0} \hat{\Phi}_{0}^{\dagger} \hat{\Phi}_{0} \hat{\rho}\right. \\
& \left.-\hat{\rho} \hat{\Phi}_{0}^{\dagger} \hat{\Phi}_{0} \hat{\Phi}_{0}^{\dagger} \hat{\Phi}_{0}\right) .
\end{aligned}
$$

Here, $\gamma_{1}$ is the damping rate of the stored qubit while $\gamma_{2}$ describes its dephasing. The two rates are given by

$$
\gamma_{1}=\frac{C \Gamma}{\Gamma^{2}+\Delta_{\text {gap }}^{2}}(1-\Xi), \quad \gamma_{2}=\frac{C}{\Gamma} \Xi,
$$

where we have introduced the dimensionless parameter $\Xi \equiv \sum_{j k} \xi_{j k} \varrho_{j}^{2} \varrho_{k}^{2} /\left(\sum_{l} \varrho_{l}^{2}\right)^{2}$ containing the spatial part of the noise correlator.

When the correlation length of the classical noise is smaller than the internuclear distance (local uncorrelated noise, $\xi_{j k} \sim \delta_{j k}$ ), $\Xi$ scales inversely with the number of nuclei (Fig. 3). In this case, the dephasing rate $\gamma_{2}$ vanishes as $1 / N$, which is an effect of the collective nature of the storage states [12]. The storage is based on encoding the logical qubit states in a large, delocalized ensemble of $N$ physical spins. As the decoherence has strongly local character, it has only a very small effect on the dephasing of the qubit. Secondly, the loss of the stored qubit is associated with a change in the number of excitations in the storage mode. Such transitions are strongly suppressed, and the damping rate $\gamma_{1}$ is decreased if $\Delta_{\text {gap }}$ is large

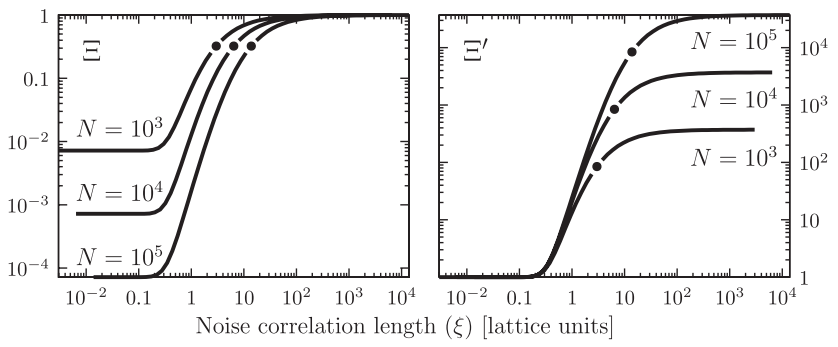

FIG. 3. The parameters $\Xi$ and $\Xi^{\prime}$ describing the effects of spatial correlations in the classical noise $\left(\xi_{j k}=e^{-r_{j k} / \xi}\right)$ for different number of nuclei. The same family of Gaussian electron densities was used as in Fig. 2. The bullets on the curves denote the linear size of the dot given by the variance $\sigma$. compared to the width of the noise spectrum $\Gamma$ (or the corresponding cutoff frequency). Finally, the opposite limit of infinite spatial correlation length $\left(\xi_{j k}=1\right)$ corresponds to a homogeneous random field resulting, e.g., from a global external source. In that case, $\Xi \approx 1$ (see Fig. 3), and there is no protection against dephasing.

Following a similar but slightly more involved procedure, we can discuss the spin-flip part $\hat{V}_{x y}=\frac{1}{2} \sum_{j}\left(B_{+}^{j} \hat{I}_{-}^{j}+\right.$ $\left.B_{-}^{j} \hat{I}_{+}^{j}\right)$ of the noise. When deriving a master equation for this case, we need to keep higher order terms in the Holstein-Primakoff approximation: in the next order, $\hat{I}_{-}^{j} \approx$ $\sqrt{2 I}\left(1-\lambda \hat{a}_{j}^{\dagger} \hat{a}_{j}\right) \hat{a}_{j}$ with $\lambda=1-(1-1 / 2 I)^{1 / 2}$. Here, we have neglected the probability of double or more excitations on the same site $j$, which is reasonable in the high polarization $(T=0)$ limit and exact for spin- $\frac{1}{2}$ nuclei. The Lindbladian describing decoherences due to spin flips reads, in leading order of $1 / N$,

$$
\begin{aligned}
\mathcal{L}_{x y}(\hat{\rho})= & \left(\gamma_{3}+\gamma_{4}\right)\left(2 \hat{\Phi}_{0} \hat{\rho} \hat{\Phi}_{0}^{\dagger}-\hat{\Phi}_{0}^{\dagger} \hat{\Phi}_{0} \hat{\rho}-\hat{\rho} \hat{\Phi}_{0}^{\dagger} \hat{\Phi}_{0}\right) \\
& +\gamma_{5}\left(2 \hat{\Phi}_{0}^{\dagger} \hat{\Phi}_{0} \hat{\rho} \hat{\Phi}_{0}^{\dagger} \hat{\Phi}_{0}-\hat{\Phi}_{0}^{\dagger} \hat{\Phi}_{0} \hat{\Phi}_{0}^{\dagger} \hat{\Phi}_{0} \hat{\rho}\right. \\
& \left.-\hat{\rho} \hat{\Phi}_{0}^{\dagger} \hat{\Phi}_{0} \hat{\Phi}_{0}^{\dagger} \hat{\Phi}_{0}\right)+\gamma_{3}\left(2 \hat{\Phi}_{0}^{\dagger} \hat{\rho} \hat{\Phi}_{0}\right. \\
& \left.-\hat{\Phi}_{0} \hat{\Phi}_{0}^{\dagger} \hat{\rho}-\hat{\rho} \hat{\Phi}_{0} \hat{\Phi}_{0}^{\dagger}\right),
\end{aligned}
$$

which describes decay with rate $\gamma_{4}$, dephasing with rate $\gamma_{5}$, and additionally thermalization (relaxation to the identity matrix) with rate $\gamma_{3}$. The rates read

$$
\begin{gathered}
\gamma_{3}=\frac{C \Gamma I \Xi^{\prime}}{\Gamma^{2}+\left(\Delta_{\text {gap }}+\Delta_{\mathrm{K}}\right)^{2}}, \quad \gamma_{4}=\frac{2 C \Gamma I \lambda^{2}}{\Gamma^{2}+\left(\Delta_{\text {gap }}-\Delta_{\mathrm{K}}\right)^{2}}, \\
\gamma_{5}=\frac{4 C \Gamma I \lambda^{2}}{\Gamma^{2}+\Delta_{\mathrm{K}}^{2}} \frac{\sum_{j} \varrho_{j}^{4}}{\left(\sum_{j} \varrho_{j}^{2}\right)^{2}} .
\end{gathered}
$$

In the limit of vanishing spatial correlations of the spin-flip noise, $\Xi^{\prime} \equiv \sum_{j k} \xi_{j k} \varrho_{j} \varrho_{k} / \sum_{l} \varrho_{l}^{2}$ tends to 1 (Fig. 3), and we have protection against thermalization $\left(\gamma_{3}\right)$ because of the separation of $|\mathbf{0}\rangle$ and $|\mathbf{1}\rangle$ by $\Delta_{\text {gap }}+\Delta_{\mathrm{K}}$. The decay corresponding to $\gamma_{4}$ is due to spin-flip induced transitions between $|\mathbf{1}\rangle$ and $\left|1_{p}, 1_{q}\right\rangle$ (the latter containing a total of two excitations but none in the storage mode), and the energy to bridge is in the order of $\Delta_{\text {gap }}-\Delta_{\mathrm{K}}$ (see Fig. 1). Finally, the last factor in the dephasing rate $\gamma_{5}$ scales as $1 / N$, indicating that it is the collective nature of the storage that leads to protection. Note that the nonlinearity of the Holstein-Primakoff representation is responsible for this dephasing: the virtual nonstorage excitations are interacting with the storage mode.

Another potential source of decoherence is nuclear-spin diffusion due to dipole-dipole interaction between nuclear spins [13]. The energy gap gives protection against this effect, too. The dipolar interaction between the pairs of spins is described in the secular approximation by

$$
\hat{H}_{D}=\sum_{j \neq k} B_{j k}\left(\hat{I}_{+}^{j} \hat{I}_{-}^{k}-2 \hat{I}_{z}^{j} \hat{I}_{z}^{k}\right) \approx 2 I \sum_{j \neq k} B_{j k} \hat{a}_{j}^{\dagger} \hat{a}_{k},
$$


where $B_{j k} \propto\left(3 \cos ^{2} \theta_{j k}-1\right) / r_{j k}^{3}, \mathbf{r}_{j k}=\mathbf{r}_{j}-\mathbf{r}_{k}$ is the distance between two nuclei, $\theta_{j k}$ is the zenith angle of the vector $\mathbf{r}_{j k}$, and we used the first order Holstein-Primakoff approximation. At full polarization, we can rewrite Eq. (9) using the bosonic spin-wave mode operators (3) as $\hat{H}_{D}=$ $\sum_{p q} \tilde{B}_{p q} \hat{\Phi}_{p}^{\dagger} \hat{\Phi}_{q}$. As if it were a central spin coupled to a mesoscopic spin bath $[14,15]$, the storage mode is coupled to a bath of nonstorage modes that present a fluctuating, effective transversal magnetic field. If the electron were not present, these fluctuations would lead, in the mean-field approximation, to a decoherence rate $\Gamma_{D} \sim$ $\left(2 \sum_{q \neq 0}\left|\tilde{B}_{0 q}\right|^{2}\right)^{1 / 2}$, which is numerically found to be in the order of $100 \mathrm{~Hz}$ for GaAs [Fig. 2(a)]. With the protective gap, however, the storage-mode operator $\hat{\Phi}_{0}$ rotates rapidly with respect to the other ones, and the above coupling averages out. The strength of the remaining coupling between the storage mode and mode $q$ is proportional to $\Delta_{\text {gap }}^{-1} \sum_{r \neq 0} \tilde{B}_{0 r} \tilde{B}_{r q}$, and the corresponding fluctuations yield a reduced decoherence rate of $\Gamma_{D}^{\prime} \sim$ $\Delta_{\text {gap }}^{-1}\left(2 \sum_{q \neq 0}\left|\sum_{r \neq 0} \tilde{B}_{0 r} \tilde{B}_{r q}\right|^{2}\right)^{1 / 2}$ as indicated in Fig. 2(a). Depending on the dot size, the effects of spin diffusion can be suppressed by several orders of magnitude.

Nonperfect nuclear-spin polarization.-It has been shown that partially polarized nuclei (at finite temperature) can also be used for storing a qubit state [4]. Instead of the fully polarized state $|\mathbf{0}\rangle$, the initial preparation drives the nuclear ensemble into a statistical mixture of dark states $\left|\mathcal{D}_{n, \beta}\right\rangle$ defined by $\hat{A}_{-}\left|\mathcal{D}_{n, \beta}\right\rangle=0$ and characterized by the total number of spins flipped $n$ and the permutation group quantum number $\beta$. As the detuning $\delta$ is adiabatically swept from far negative to far positive, a superposition of the $|\downarrow\rangle_{e}$ and $|\uparrow\rangle_{e}$ electron spin states is mapped into the mixture of superpositions of the nuclear-spin states $\left|\mathcal{D}_{n, \beta}\right\rangle$ and $\left|\mathcal{E}_{n, \beta}\right\rangle \equiv \frac{\mathcal{A}}{\Omega_{n}} \hat{A}_{+}\left|\mathcal{D}_{n, \beta}\right\rangle$, and the qubit state is efficiently written into the memory [4].

When the electron is left in the quantum dot, it feels different Overhauser fields for different dark states; hence, the detuning should be adjusted such that $\overline{\delta_{n}^{\mathrm{OH}}+\delta^{\mathrm{el}}} \gg$ $\operatorname{Var}\left(\delta_{n}^{\mathrm{OH}}\right)$. Moreover, the hyperfine Rabi frequency $\Omega_{n}$ also varies with $n$ and the energy gap $\Delta_{\text {gap }, n}$ is not the same for all dark states. This inhomogeneous broadening would result in dephasing of the qubit, but can be avoided by a symmetric spin echo sequence [4].

Because of the inhomogeneous nature of the hyperfine coupling, the $\hat{A}_{z, \pm}$ operators do not follow the angular momentum commutation relation. Therefore, $\left|\mathcal{D}_{n, \beta}\right\rangle$ is not an eigenstate of the Knight shift operator, but it is partially mapped into an orthogonal state: $\hat{H}_{\mathrm{K}}\left|\mathcal{D}_{n, \beta}\right\rangle=$ $-\frac{1}{2} \delta_{n}^{\mathrm{OH}}\left|\mathcal{D}_{n, \beta}\right\rangle+\omega_{n}\left|\mathcal{D}_{n, \beta}^{\perp}\right\rangle$. Furthermore, $\left|\mathcal{E}_{n, \beta}\right\rangle$ is neither an eigenstate of $\hat{H}_{\text {gap }}$ nor of $\hat{H}_{\mathrm{K}}: \hat{H}\left|\mathcal{E}_{n, \beta}\right\rangle=\left(-\frac{1}{2} \delta_{n}^{\mathrm{OH}}+\right.$ $\left.\Delta_{\mathrm{K}, n}+\Delta_{\text {gap }, n}\right)\left|\mathcal{E}_{n, \beta}\right\rangle+\zeta_{n}\left|\mathcal{E}_{n, \beta}^{\perp}\right\rangle$. The parameters can be expressed as expectation values in $\left|\mathcal{D}_{n, \beta}\right\rangle$ :

$$
\begin{gathered}
\Omega_{n}^{2}=\mathcal{A}^{2}\left\langle\hat{A}_{-} \hat{A}_{+}\right\rangle, \quad \omega_{n}^{2}=\frac{\mathcal{A}^{2}}{4}\left(\left\langle\hat{A}_{z}^{2}\right\rangle-\left\langle\hat{A}_{z}\right\rangle^{2}\right), \\
\Delta_{\text {gap }, n}=\mathcal{A}^{4}\left\langle\hat{A}_{-} \hat{A}_{+} \hat{A}_{-} \hat{A}_{+}\right\rangle / 4 \delta_{n} \Omega_{n}^{2}, \\
\Delta_{\mathrm{K}, n}=\frac{\mathcal{A}}{2}\left\langle\hat{A}_{z}\right\rangle-\mathcal{A}^{3}\left\langle\hat{A}_{-} \hat{A}_{z} \hat{A}_{+}\right\rangle / 2 \Omega_{n}^{2} \\
\zeta^{2}=\left\langle\mathcal{E}_{n, \beta}\left|\hat{H}^{2}\right| \mathcal{E}_{n, \beta}\right\rangle-\left\langle\mathcal{E}_{n, \beta}|\hat{H}| \mathcal{E}_{n, \beta}\right\rangle^{2} .
\end{gathered}
$$

The explicit form of the inhomogeneous dark states [4] allows us to estimate these values [see Fig. 2(b)]. We expect that the storage mode is still protected as long as $\omega_{n}$ and $\zeta_{n}$ are much smaller than $\Delta_{\text {gap }, n}$. Our simulation suggests that even for a polarization of $80 \%\left(n=10^{4}\right)$, the gap is more than 5 times larger than $\omega_{n}$ and $\zeta_{n}$.

In summary, we have demonstrated the suppression of spin dephasing and spin flips in a quantum memory consisting of a delocalized ensemble of nuclear spins in a quantum dot if the noise has a highly local character and the spectral width of the noise spectrum is small compared to the energy gap. We have shown that the memory can be protected against nuclear-spin diffusion mediated by dipole-dipole interaction. We have also analyzed the effects of inhomogeneous hyperfine couplings and imperfect initial nuclear-spin polarization.

J.M. T. wishes to thank the Fleischhauer group for their kind hospitality during his stay. This work was supported by the EU MRTN-CT-2006-035369 network EMALI; J. M. T. is supported by Pappalardo.

*Current address: Department of Physics and Astronomy, University of Aarhus, DK-8000 Århus C, Denmark

[1] A. Imamoglu et al., Phys. Rev. Lett. 91, 017402 (2003).

[2] H. Christ, J. I. Cirac, and G. Giedke, Phys. Rev. B 75, 155324 (2007).

[3] J. M. Taylor, C. M. Marcus, and M. D. Lukin, Phys. Rev. Lett. 90, 206803 (2003).

[4] J. M. Taylor, A. Imamoglu, and M. D. Lukin, Phys. Rev. Lett. 91, 246802 (2003).

[5] H. Schwager, J. I. Cirac, and G. Giedke, arXiv:0810.4488; arXiv:0903.1727.

[6] M. V. Gurudev Dutt et al., Science 316, 1312 (2007).

[7] P. Neumann et al., Science 320, 1326 (2008).

[8] V. V. Dobrovitski, J. M. Taylor, and M. D. Lukin, Phys. Rev. B 73, 245318 (2006).

[9] C. W. Lai et al., Phys. Rev. Lett. 96, 167403 (2006).

[10] G. Giedke et al., Phys. Rev. A 74, 032316 (2006).

[11] J.A. Gupta and D.D. Awschalom, Phys. Rev. B 63, 085303 (2001).

[12] C. Mewes and M. Fleischhauer, Phys. Rev. A 72, 022327 (2005); C. Mewes, Ph.D. thesis, University of Kaiserslautern, Germany, 2002.

[13] C. Deng and X. Hu, IEEE Trans. Nanotechnol. 4, 35 (2005).

[14] A. V. Khaetskii, D. Loss, and L. Glazman, Phys. Rev. Lett. 88, 186802 (2002); Phys. Rev. B 67, 195329 (2003).

[15] W. M. Witzel and S. Das Sarma, Phys. Rev. B 74, 035322 (2006). 